\title{
Note on a paper by Joung Min Song
}

\author{
by \\ Gérald Tenenbaum (Nancy)
}

In [3], Joung Min Song established fairly precise estimates for weighted sums of the form

$$
\sum_{\substack{n \leqslant x \\ P(n) \leqslant y}} \frac{h(n)}{n},
$$

where $P(n)$ denotes the largest prime factor of an integer $n$ with the convention that $P(1)=1$, and $h$ is a non-negative multiplicative arithmetic function satisfying the conditions

$$
\begin{aligned}
\sum_{p \leqslant z}\{h(p)-\kappa\} \frac{\log p}{p} \ll(\log z)^{1-\delta} \quad(z \geqslant 2), \\
\sum_{p, \nu \geqslant 2} \frac{h\left(p^{\nu}\right) \log p^{\nu}}{p^{\nu}}<\infty,
\end{aligned}
$$

with suitable constants $\kappa>0, \delta \in] 0,1[$.

Song's proof, which rests on an elegant theorem of Halberstam corresponding to the case $y \geqslant x$, is a nice development of Wirsing's ideas in [5]. A handy feature of this result is that, apart from positivity, only a mild average assumption is made upon the values $h(p)$.

A natural alternative approach to this problem is to first establish the result for some special arithmetic function satisfying $h(p)=\kappa$ for all $p$, for which much more information is available, and then derive the general statement from a suitable comparison result.

The main purpose of this note is to show that such a strategy is indeed successful. For fixed positive $\kappa$, we let $\tau_{\kappa}(n)$ be the coefficient of $1 / n^{s}$ in the Dirichlet series expansion of $\zeta(s)^{\kappa}$, where $\zeta(s)$ denotes the Riemann zeta function. We shall select $n \mapsto \tau_{\kappa}(n)$ to play the rôle of the special function mentioned above. As we shall see, Smida's results in [2] almost

2000 Mathematics Subject Classification: Primary 11N37; Secondary 11N25, 11N60. 
readily provide the required estimates, and so we shall mainly be concerned with the comparison result.

Put

$$
V_{h}(y):=\prod_{p \leqslant y} \sum_{\nu \geqslant 0} h\left(p^{\nu}\right) / p^{\nu} .
$$

Furthermore, define, as in [3], the function $j_{\kappa}$ as the continuous solution to the differential-difference equation

$$
u j_{\kappa}^{\prime}(u)-\kappa j_{\kappa}(u)+\kappa j_{\kappa}(u-1)=0 \quad(u>1)
$$

with initial conditions $j_{\kappa}(u):=0(u<0)$ and $j_{\kappa}(u):=\mathrm{e}^{-\gamma \kappa} u^{\kappa} / \Gamma(\kappa+1)$ $(0 \leqslant u \leqslant 1)$, where $\gamma$ is Euler's constant. We prove the following result.

THEOREM. Let $h$ be a non-negative multiplicative arithmetic function. Under assumptions $\left(\Omega_{1}\right)$ and $\left(\Omega_{2}\right)$, we have

$$
\sum_{\substack{n \leqslant x \\ P(n) \leqslant y}} \frac{h(n)}{n}=V_{h}(y) j_{\kappa}(u)\left\{1+O\left(\frac{1}{(\log y)^{\delta}}\right)\right\}
$$

uniformly for $x \geqslant y \geqslant 2$ and with $u:=(\log x) / \log y$. Furthermore, the same formula holds for all complex multiplicative functions $h$ satisfying

$$
\begin{gathered}
\sum_{p \leqslant z} \frac{|h(p)-\kappa| \log p}{p} \ll(\log z)^{1-\delta} \quad(z \geqslant 2), \\
\sum_{p, \nu \geqslant 2} \frac{\left|h\left(p^{\nu}\right)\right| \log p^{\nu}}{p^{\nu}}<\infty \\
\sum_{\nu \geqslant 0} \frac{h\left(p^{\nu}\right)}{p^{\nu}} \neq 0 \quad(p \geqslant 2),
\end{gathered}
$$

for suitable constants $\kappa>0, \delta \in] 0,1[$.

As described above, this result is essentially proved by first showing that it holds for $h=\tau_{\kappa}$ and then extending the formula to the stated hypotheses. However, it should be stressed that Halberstam's theorem mentioned above is crucial for the initial step $x \leqslant y$. Without this, using for instance Wirsing's theorem [5], the method would require slightly stronger hypotheses upon $h$, e.g. Wirsing's conditions $h\left(p^{\nu}\right) \leqslant \gamma_{1} \gamma_{2}^{\nu}$ with $\gamma_{1}>0,0<\gamma_{2}<2$, and the size of the error term would be regulated by available effective forms of Wirsing's theorem. $\left({ }^{1}\right)$

$\left({ }^{1}\right)$ For instance the effective form of Karamata's theorem given in [4], Theorem II.7.9, would give an error factor $1+O\left(1 / \log _{2} 2 y\right)$. This is much weaker than desired, but Karamata's theorem is "too" general a tool for this application: it deals with Dirichlet series whose coefficients need not be multiplicative. Following Wirsing, Halberstam's proof strongly exploits multiplicativity and the calculations required to obtain the sharp effective estimate are comparatively simple. 
Formula (1) is slightly more precise than Song's. Since $j_{\kappa}(u)$ tends very quickly towards 1 , it is significant only in a restricted range for $y$, certainly included in the domain $u \leqslant \log _{2} y$. $\left({ }^{2}\right)$ This is to be expected because the information on $h$ is roughly equivalent to one regarding the corresponding Dirichlet series in a small neighbourhood (of size depending on $y$ ) of the point $s=1$ and, as shown by saddle point analysis, a larger range would involve information in the half-plane $\operatorname{Re} s<1$ outside this neighbourhood. In turn, such assumptions essentially amount to controlling averages of the form $\sum_{p \leqslant z} h(p) / p^{\alpha}$ with some $\alpha<1$. When, for instance, $1-\alpha$ is bounded away from 0 , this is equivalent to controlling $\sum_{p \leqslant z} h(p)$.

We finally note that hypothesis $\left(\Omega_{3}^{*}\right)$ is only necessary to fit the specific formulation given in (1). Indeed, without this assumption we obtain

$$
\sum_{\substack{n \leqslant x \\ P(n) \leqslant y}} \frac{h(n)}{n}=V_{h}(y) j_{\kappa}(u)+O\left((\log y)^{\kappa-\delta}\right) .
$$

Let $z_{\kappa}$ denote the unique solution of the differential-difference equation

$$
\begin{cases}u z_{\kappa}^{\prime}(u)+\kappa z_{\kappa}(u-1)=0 & (u>1), \\ z_{\kappa}(u)=1 & (0 \leqslant u \leqslant 1), \\ z_{\kappa}(u)=0 & (u<0),\end{cases}
$$

which is continuous on $[0, \infty[$. Then a simple calculation shows that, for all real numbers $u$, we have

$$
\frac{1}{\Gamma(\kappa)} \int_{0}^{u} v^{\kappa-1} z_{\kappa}(u-v) \mathrm{d} v=\mathrm{e}^{\gamma \kappa} j_{\kappa}(u)=\int_{0}^{u} \varrho_{\kappa}(v) \mathrm{d} v,
$$

where $\varrho_{\kappa}$ is the $\kappa$ th fractional convolution power of the Dickman function precisely defined in Smida's paper [2]. Writing

$$
S_{\kappa}(x):=\sum_{n \leqslant x} \tau_{\kappa}(n)
$$

Smida showed in [2] that, for any fixed $\varepsilon>0$,

$$
\begin{aligned}
S_{\kappa}(x, y):= & \sum_{\substack{n \leqslant x \\
P(n) \leqslant y}} \tau_{\kappa}(n) \\
& =\left\{1+O\left(\frac{1}{L_{\varepsilon}(y)}\right)\right\} x \int_{0-}^{\infty} z_{\kappa}(u-v) \mathrm{d}\left(\frac{S_{\kappa}\left(y^{v}\right)}{y^{v}}\right)
\end{aligned}
$$

holds uniformly, with $u:=(\log x) / \log y, L_{\varepsilon}(y):=\mathrm{e}^{(\log y)^{3 / 5-\varepsilon}}$, in the domain

$$
x \geqslant 3, \quad \mathrm{e}^{(\log x)^{2 / 5+\varepsilon}} \leqslant y \leqslant x .
$$

$\left({ }^{2}\right)$ Here and throughout the paper, we let $\log _{k}$ denote the $k$-fold iterated logarithm. 
She also proved that, for fixed $\kappa>0, \varepsilon>0$,

$$
S_{\kappa}(x, y)=x(\log y)^{\kappa-1} \varrho_{\kappa}(u)\left\{1+O\left(\frac{\log (u+1)}{\log y}+\frac{1}{(\log y)^{\kappa}}\right)\right\}
$$

uniformly for $x \geqslant 3, \mathrm{e}^{\left(\log _{2} x\right)^{5 / 3+\varepsilon}} \leqslant y \leqslant x$.

We shall deduce our theorem mainly from (5) and (7).

To prove (1), we first investigate the case $h=\tau_{\kappa}$. For notational simplicity, we write $V_{\kappa}(y)$ instead of $V_{\tau_{\kappa}}(y)$. We shall show that the estimate

$$
V_{\kappa}(x, y):=\sum_{\substack{n \leqslant x \\ P(n) \leqslant y}} \frac{\tau_{\kappa}(n)}{n}=V_{\kappa}(y) j_{\kappa}(u)\left\{1+O\left(\frac{\log _{3} 8 y}{\log y}\right)\right\}
$$

holds uniformly for $x \geqslant y \geqslant 2$, with

$$
V_{\kappa}(y):=\prod_{p \leqslant y}\left(1-\frac{1}{p}\right)^{-\kappa}=\left\{1+O\left(\frac{1}{L_{\varepsilon}(y)}\right)\right\} \mathrm{e}^{\gamma \kappa}(\log y)^{\kappa} .
$$

We may assume that (6) holds, and indeed that $u \leqslant \log _{2} y$, since otherwise $j_{\kappa}(u)=1+O(1 / \log y)$ and the left-hand side of (8) is a non-decreasing function of $u$. By partial summation

$$
\sum_{\substack{n \leqslant x \\ P(n) \leqslant y}} \frac{\tau_{\kappa}(n)}{n}=\int_{1}^{x} \frac{S_{\kappa}(t, y)}{t^{2}} \mathrm{~d} t+\frac{S_{\kappa}(x, y)}{x} .
$$

By $(7)$, the second term on the right is $\ll(\log y)^{\kappa-1}$, and so may be absorbed by the error term in (8). To estimate the first term, we consider two cases, according as $\kappa \geqslant 1$ or not.

When $\kappa \geqslant 1$, we have from (7), in the considered range,

$$
\int_{1}^{x} \frac{S_{\kappa}(t, y)}{t^{2}} \mathrm{~d} t=\left\{1+O\left(\frac{\log _{3} 8 y}{\log y}\right)\right\}(\log y)^{\kappa} \int_{0}^{u} \varrho_{\kappa}(v) \mathrm{d} v,
$$

which implies (8) by (4) and (9).

When $0<\kappa<1$, we apply (5) and reverse the order of summations. We obtain

$$
\int_{1}^{x} \frac{S_{\kappa}(t, y)}{t^{2}} \mathrm{~d} t=\left\{1+O\left(\frac{1}{L_{\varepsilon}(y)}\right)\right\} J_{\kappa}(x, y)
$$

with

$$
J_{\kappa}(x, y):=\int_{0-}^{\infty} \mathrm{d}\left(\frac{S_{\kappa}\left(y^{v}\right)}{y^{v}}\right) \int_{1}^{x} z_{\kappa}\left(\frac{\log t}{\log y}-v\right) \frac{\mathrm{d} t}{t}
$$




$$
=(\log y) \int_{0-}^{\infty} \mathrm{d}\left(\frac{S_{\kappa}\left(y^{v}\right)}{y^{v}}\right) \int_{0}^{u} z_{\kappa}(w-v) \mathrm{d} w=(\log y) \int_{0}^{u} z_{\kappa}(u-v) \frac{S_{\kappa}\left(y^{v}\right)}{y^{v}} \mathrm{~d} v .
$$

Using the classical estimate

$$
\begin{aligned}
(\log y) \int_{0}^{u} \frac{S_{\kappa}\left(y^{v}\right)}{y^{v}} \mathrm{~d} v & =\sum_{n \leqslant x} \frac{\tau_{\kappa}(n)}{n}-\frac{1}{x} \sum_{n \leqslant x} \tau_{\kappa}(n) \\
& =\frac{(u \log y)^{\kappa}}{\Gamma(\kappa+1)}+O\left(\frac{(u \log y)^{\kappa}}{1+u \log y}\right),
\end{aligned}
$$

valid uniformly for $y \geqslant 2, u \geqslant 0$, we derive from the above and (4) the formula

$$
\begin{aligned}
J_{\kappa}(x, y) & =\frac{(\log y)^{\kappa}}{\Gamma(\kappa)} \int_{0}^{u} v^{\kappa-1} z_{\kappa}(u-v) \mathrm{d} v+R \\
& =j_{\kappa}(u) \mathrm{e}^{\gamma \kappa}(\log y)^{\kappa}+R
\end{aligned}
$$

with

$$
R=\int_{0}^{u} z_{\kappa}(u-v) \mathrm{d}\left\{O\left(\frac{(v \log y)^{\kappa}}{1+v \log y}\right)\right\} .
$$

This quantity may be estimated by partial summation, admitting for the moment that, still for $0<\kappa<1$,

$$
z_{\kappa}^{\prime}(v) \leqslant 0 \quad(v>0, v \neq 1) .
$$

Indeed, we obtain, conditionally to (12), for $u \geqslant 1$,

$$
R \ll(\log y)^{\kappa-1}-(\log y)^{\kappa-1} \int_{0}^{u} z_{\kappa}^{\prime}(u-v) v^{\kappa-1} \mathrm{~d} v \ll(\log y)^{\kappa-1},
$$

where the last integral has been estimated by differentiating (4).

It remains to establish (12). We first note that (3) implies

$$
\left\{u^{\kappa} z_{\kappa}(u)\right\}^{\prime}=\kappa u^{\kappa-1}\left\{z_{\kappa}(u)-z_{\kappa}(u-1)\right\} \quad(u>0, u \neq 1)
$$

and so, for all $u \geqslant 1$,

$$
u^{\kappa} z_{\kappa}(u)=\kappa \int_{0}^{u-1}\left\{v^{\kappa-1}-(v+1)^{\kappa-1}\right\} z_{\kappa}(v) \mathrm{d} v+\kappa \int_{u-1}^{u} v^{\kappa-1} z_{\kappa}(v) \mathrm{d} v .
$$

This plainly implies that $z_{\kappa}(u) \geqslant 0$ for all $u$ and (12) follows in view of (3). This completes the proof of (8). 
We now prove the first assertion of our theorem. Let $V_{h}(x, y)$ denote the sum on the left-hand side of (1). As in Song's paper, we introduce

$$
T_{h}(x, y):=\int_{1}^{x} V_{h}(t, y) \frac{\mathrm{d} t}{t}=\sum_{\substack{n \leqslant x \\ P(n) \leqslant y}} \frac{h(n)}{n} \log \left(\frac{x}{n}\right) .
$$

Wirsing's functional equation for $V_{h}(x, y)$ stated and proved in Lemma 2 of [3] readily provides (see equation (4.12) of [3])

$$
V_{h}(x, y) \log x=(\kappa+1) T_{h}(x, y)-\kappa T_{h}(x / y, y)+O\left((\log y)^{\kappa+1-\delta}\right) .
$$

We consider $y$ as fixed and introduce

$$
\lambda_{h}(u):=T_{h}(x, y) /(\log y)^{\kappa+1}=T_{h}\left(y^{u}, y\right) /(\log y)^{\kappa+1} .
$$

This function of $u$ is differentiable except perhaps when $y^{u} \in \mathbb{N}$. At these points, we define $\lambda_{h}^{\prime}(u)$ by right continuity, so that $\lambda_{h}^{\prime}(u)=V_{h}\left(y^{u}, y\right) /(\log y)^{\kappa}$ for all $u$. Then (13) can be rewritten as

$$
u \lambda_{h}^{\prime}(u)=(\kappa+1) \lambda_{h}(u)-\kappa \lambda_{h}(u-1)+O\left(\frac{1}{(\log y)^{\delta}}\right) \quad(u \geqslant 1) .
$$

We apply this for both functions $h$ and $\tau_{k}$, multiply the second equation by

$$
C_{\kappa}(h):=\prod_{p}\left(1-\frac{1}{p}\right)^{\kappa} \sum_{\nu \geqslant 0} \frac{h\left(p^{\nu}\right)}{p^{\nu}}
$$

and subtract. Writing $\mu(u):=\lambda_{h}(u)-C_{\kappa}(h) \lambda_{\tau_{\kappa}}(u)$, we get

$$
u \mu^{\prime}(u)=(\kappa+1) \mu(u)-\kappa \mu(u-1)+O\left(\frac{1}{(\log y)^{\delta}}\right) \quad(u \geqslant 1),
$$

which can be rewritten as

$$
\frac{\mathrm{d}}{\mathrm{d} u}\left\{\frac{\mu(u)}{u^{\kappa+1}}\right\}=-\kappa \frac{\mu(u-1)}{u^{\kappa+2}}+O\left(\frac{1}{u^{\kappa+2}(\log y)^{\delta}}\right) \quad(u \geqslant 1),
$$

from which we infer that, for $1 \leqslant u \leqslant v \leqslant u+1$,

$$
\frac{|\mu(v)|}{v^{\kappa+1}} \leqslant \frac{|\mu(u)|}{u^{\kappa+1}}+\kappa \int_{u}^{v} \frac{|\mu(w-1)|}{w^{\kappa+2}} \mathrm{~d} w+O\left(\frac{1}{v^{\kappa+2}(\log y)^{\delta}}\right) .
$$

We now observe that Halberstam's theorem stated and proved in [3] (Theorem A), implies

$$
\mu(u) \ll 1 /(\log y)^{\delta}
$$

for $0 \leqslant u \leqslant 1$. Thus, by a routine induction, (15) yields that (16) remains true for bounded $u$. Let $q:] 0, \infty[\rightarrow \mathbb{R}$ be the solution defined in [1] to the adjoint equation corresponding to (14), namely

$$
u q^{\prime}(u)+(\kappa+2) q(u)-\kappa q(u+1)=0 \quad(u>0) .
$$


Then $q(u) \sim 1 / u^{2}$ as $u \rightarrow \infty$. Moreover, from (14) and (17) we get

$$
\frac{\mathrm{d}}{\mathrm{d} u}\left\{u \mu(u) q(u)-\kappa \int_{u-1}^{u} \mu(v) q(v+1) \mathrm{d} v\right\} \ll \frac{q(u)}{(\log y)^{\delta}} \quad(u \geqslant 1) .
$$

We integrate this over the range $[1, u]$ and use (16) for $0 \leqslant u \leqslant 1$. We obtain

$$
u \mu(u) q(u)=\kappa \int_{u-1}^{u} \mu(v) q(v+1) \mathrm{d} v+O\left(\frac{1}{(\log y)^{\delta}}\right) \quad(u \geqslant 1) .
$$

We use this formula in the form that, for a suitable constant $A>0$, we have

$$
u|\mu(u) q(u)| \leqslant \kappa \int_{u-1}^{u}|\mu(v) q(v+1)| \mathrm{d} v+\frac{A}{(\log y)^{\delta}} \quad(u \geqslant 1) .
$$

We are now in a position to conclude the argument. Let $u_{0}>8 \kappa+1$ be such that $\frac{1}{2} \leqslant q(u) u^{2} \leqslant 2$ for $u \geqslant u_{0}$, and let $B$ denote a constant exceeding $4 A$ and such that

$$
|\mu(u)| \leqslant B u /(\log y)^{\delta} \quad\left(1 \leqslant u \leqslant u_{0}\right)
$$

Define $u_{1}$ as the greatest lower bound of values of $u$ with $|\mu(u)|>B u /(\log y)^{\delta}$. Then, trivially, $u_{1} \geqslant u_{0}$. Moreover, if $u_{1}$ is finite, then by (18) we have

$$
\frac{1}{2} B \leqslant 2 \kappa B / u_{1}+A \text {. }
$$

This in turn implies $\frac{1}{2}-2 \kappa / u_{1} \leqslant A / B \leqslant \frac{1}{4}$, whence $u_{1} \leqslant 8 \kappa$, a contradiction. Thus, $u_{1}$ is not finite and we have

$$
|\mu(u)| \leqslant B u /(\log y)^{\delta} \quad(u \geqslant 1) .
$$

Inserting back in (14), using (8) and the fact that

$$
C_{\kappa}(h)=\prod_{p \leqslant y}\left(1-\frac{1}{p}\right)^{\kappa} \sum_{\nu \geqslant 0} \frac{h\left(p^{\nu}\right)}{p^{\nu}}\left\{1+O\left(\frac{1}{(\log y)^{\delta}}\right)\right\},
$$

which easily follows from $\left(\Omega_{1}\right)$ and $\left(\Omega_{2}\right)$, we obtain (1).

We now prove the second part of our theorem. A standard convolution argument yields $(1)$ from $(8)$ under assumptions $\left(\Omega_{j}^{*}\right)(1 \leqslant j \leqslant 3)$. Indeed, writing $h=\tau_{\kappa} * g$, we deduce in turn from $\left(\Omega_{1}^{*}\right)$ and $\left(\Omega_{2}^{*}\right)$ that

$$
\sum_{p, \nu \geqslant 2}\left|g\left(p^{\nu}\right)\right| / p^{\nu}<\infty, \quad \sum_{m \geqslant 1}|g(m)| / m<\infty
$$

and that

$$
\begin{aligned}
\sum_{P(n) \leqslant y} \frac{|g(n)| \log n}{n} & \leqslant \sum_{P(m) \leqslant y} \frac{|g(m)|}{m} \sum_{p \leqslant y, \nu \geqslant 1} \frac{\left|g\left(p^{\nu}\right)\right|}{p^{\nu}} \log p^{\nu} \\
& \ll(\log y)^{1-\delta} .
\end{aligned}
$$


Now

$$
\sum_{\substack{n \leqslant x \\ P(n) \leqslant y}} \frac{h(n)}{n}=\sum_{\substack{d \leqslant x \\ P(d) \leqslant y}} \frac{g(d)}{d} V_{\kappa}(x / d, y)
$$

and, using the fact that $j_{\kappa}(u)=j_{\kappa}(1) u^{\kappa}$ for $0 \leqslant u \leqslant 1$, we deduce from (8) and classical estimates for $V_{\kappa}(y, y)$ that

$$
V_{\kappa}(x / d, y)=j_{\kappa}(u) V_{\kappa}(y)\left\{1+O\left(\frac{\log _{3} 8 y+\log d}{\log y}\right)\right\}
$$

uniformly for $x \geqslant y \geqslant 2, d \geqslant 1$. Taking (19) into account, we thus obtain

$$
\begin{gathered}
\sum_{\substack{n \leqslant x \\
P(n) \leqslant y}} \frac{h(n)}{n}=V_{\kappa}(y) \sum_{\substack{d \leqslant x \\
P(d) \leqslant y}} \frac{g(d)}{d} j_{\kappa}(u)\left\{1+O\left(\frac{\log _{3} 8 y+\log d}{\log y}\right)\right\} \\
=V_{\kappa}(y) j_{\kappa}(u)\left\{\sum_{P(d) \leqslant y} \frac{g(d)}{d}+O\left(\sum_{P(d) \leqslant d} \frac{|g(d)|\left(\log _{3} 8 y+\log d\right)}{d \log y}\right)\right\} \\
=V_{h}(y) j_{\kappa}(u)\left\{1+O\left(\frac{1}{(\log y)^{\delta}}\right)\right\} .
\end{gathered}
$$

This completes the proof of (1).

\section{References}

[1] H. Iwaniec, Rosser's sieve, Acta Arith. 36 (1980), 171-202.

[2] H. Smida, Valeur moyenne des fonctions de Piltz sur les entiers sans grand facteur premier, ibid. 63 (1993), 21-50.

[3] J. M. Song, Sums of nonnegative multiplicative functions over integers without large prime factors $I$, this issue, 329-351.

[4] G. Tenenbaum, Introduction to Analytic and Probabilistic Number Theory, Cambridge Stud. Adv. Math. 46, Cambridge Univ. Press, 1995.

[5] E. Wirsing, Das asymptotische Verhalten von Summen über multiplikative Funktionen, Math. Ann. 143 (1961), 75-102.

Institut Élie Cartan

Université Henri Poincaré-Nancy 1

BP 239

54506 Vandœuvre Cedex, France

E-mail: tenenb@ciril.fr 